\title{
UMA COMPARAÇÃO DE PRÁTICAS EXPLANATÓRIAS DO MENTALISMO E DA ANÁLISE DO COMPORTAMENTO
}

\section{A COMPARISON OF THE EXPLANATORY PRACTICES OF MENTALISM AND BEHAVIOR ANALYSIS}

\author{
JAY MOORE \\ UNIVERSITY OF WISCONSIN-MILWAUKEE, ESTADOS UNIDOS
}

\section{RESUMO}

O mentalismo é uma orientação à explicação causal do comportamento em que as causas são inferidas como estruturas não observáveis, pertencentes a domínios não comportamentais. Tipicamente, essas estruturas são tidas como subjacentes ao comportamento, e o domínio em questão é o da "mente". Em alguns casos, com algumas exceções, o mentalismo dialoga com a psicologia tradicional ou com o dualismo de substância. Os argumentos em prol das explicações mentais subscritos aos níveis teórico e conceitual não levam em consideração a fonte das referidas explicações. A análise do comportamento se opõe ao mentalismo por razões pragmáticas, ao invés de ontológicas: o mentalismo impede uma análise do comportamento genuína, que possa contribuir no âmbito da previsão e do controle, por confundir os cientistas ao induzi-los a aceitar explicações inefetivas acerca do seu objeto de estudo.

Palavras-chave: análise do comportamento, explicação, mentalismo, método científico, comportamento verbal

\begin{abstract}
Mentalism is an orientation to the causal explanation of behavior in which the causes are inferred to be unobservable structures from a non-behavioral domain. Typically, the structures are held to underlie behavior, and the domain is that of "mind." In some but not all cases, mentalism subscribes to traditional psychophysical or substance dualism. Arguments that mental explanations are at the theoretical or conceptual level fail to consider the source of the explanation in question. Behavior analysts oppose mentalism on pragmatic, rather than ontological grounds: mentalism impedes a genuine science of behavior contributing to prediction and control by misleading scientists and inducing them to accept ineffective explanations of their subject matter.
\end{abstract}

Key words: behavior analysis, explanation, mentalism, scientific method, theory, verbal behavior

\footnotetext{
Esse artigo é produto de material que desenvolvi ao longo dos anos para auxiliar em meu próprio ensino sobre as práticas explanatórias do mentalismo e da análise do comportamento. Eu o apresento aqui na expectativa de que outros o considerem útil. Assegurando o objetivo puramente instrucional do artigo, utilizei o menor número possível de referências. Além disso, a linguagem bem como a apresentação da argumentação, são mais informais do que em outros artigos. Peço desculpas e tolerância por parte dos leitores caso a execução do mesmo tenha ficado aquém do esperado. Posso apenas dizer que sou um produto inacabado de contingências que ainda atuam sobre mim.

Correspondências sobre esse material devem ser encaminhadas ao e-mail do autor jcm@uwm.edu, ou para sua residência: 1861 E Fox Lane; Fox Point, WI 53217; USA.

Tradução: Marcelo V. Silveira (Pós-doutorando em Neurociências na Universidade Federal do ABC - Bolsista FAPESP \#2017/06178-

7 ) e César Antônio Alves da Rocha (discente do Programa de Pós-Graduação em Psicologia, Universidade Federal de São Carlos Bolsista FAPESP \# 2014/02981-1).
} 


\section{UMA COMPARAÇÃO DE PRÁTICAS EXPLANATÓRIAS DO MENTALISMO E DA ANÁLISE DO COMPORTAMENTO}

O Mentalismo é a orientação dominante na psicologia contemporânea subscrita às explicações causais do comportamento. O mentalismo pode assumir muitas formas. Desde (a) o desenvolvimento da personalidade e psicopatologia, sob o ponto de vista da psicanálise Freudiana; (b) psicologia cognitiva contemporânea e processamento de informação; (c) psicologia social contemporânea; (d) modelos médicos contemporâneos para a patologia comportamental, conforme o DSM-V. O presente artigo revisa as características principais do mentalismo e compara suas práticas explanatórias com as práticas da análise do comportamento. Devido ao incontável número de posições mentalistas, a presente revisão é, em muitas instâncias, necessariamente abstrata, porém, com ajustes que possam atender a demandas específicas, ela é aplicável a casos particulares.

\section{UMA DEFINIÇÃO DE MENTALISMO}

Falamos que uma explicação é mentalista quando ela apresenta as seguintes características:

- Que o principal objetivo da psicologia como ciência é postular um conjunto de atos, estados, mecanismos, processos, entidades e estruturas cujas propriedades funcionais possibilitam qualquer forma de comportamento. Usaremos, de agora para diante, apenas um termo: estruturas.

- Que tais estruturas estejam em um aparato psicológico que é intrínseco ao organismo. Um organismo não as adquire ao longo de sua vida por meio da experiência com seu meio ambiente. Elas podem, por exemplo, ser desenvolvimentais ou evolucionárias.

- Medidas fisiológicas fornecem correlatos neurais e evidências das estruturas mentais subjacentes, mas não as definem. As estruturas são definidas por suas propriedades funcionais, tais como capacidades, conteúdos, janela de tempo para processamento de informação, e outras, e não suas funcionalidades físicas ou ocorrências observáveis. As estruturas dão substância às competências que favorecem o comportamento observável do organismo em qualquer situação em que ele se encontre. Por último, o organismo que se comporta não é necessariamente consciente do funcionamento dessas estruturas mentais.

- As estruturas são inferidas e são subjacentes, ao invés de observáveis.

- As estruturas e suas propriedades podem ser tomadas como causas e a especificação das estruturas, de suas propriedades funcionais e da arquitetura do sistema como um todo, até mesmo em nível conceitual, integram a explicação causal para o comportamento.

O presumido locus dessas estruturas varia nas diferentes versões do mentalismo. Em algumas versões, infere-se que as estruturas estão, de algum modo, dentro do organismo, talvez como manifestações de estruturas situadas no córtex cerebral. Em outros casos, infere-se que elas existem em um nível conceitual, assim seria desnecessário colocar em questão qual é o locus da estrutura, ou propriedade, que foi inferida, do mesmo modo que é desnecessário colocar em questão a localização da força em cavalos no interior de um motor de combustão interna. Todavia, inúmeras formas de dados observáveis (i.e., evidências) são utilizadas de modo a dar sustentação ao projeto inferencial. Esses dados podem ser de natureza comportamental (tempo de reação, rastreamento do movimento dos olhos, raciocínio, julgamentos perceptuais) ou fisiológicos (fMRI, PET, potenciais de ação e mecanismos sinápticos em estruturas ou circuitos corticais). Neste particular, o comportamento observável é relevante porque oferece evidência que dá suporte às inferências sobre as propriedades causais das estruturas mentais, em vez de ser ele próprio o objeto a ser estudado.

Novamente, o mentalismo assume de modo implícito que o aparato psicológico do organismo estaria para além do domínio no qual o comportamento acontece. O domínio de uma explicação é preocupante quando seus conceitos analíticos e seus métodos de observação estão desvinculados dos fatos relativos ao comportamento, os quais eles dizem explicar. Mental, cognitivo, subjetivo, espiritual, psíquico, conceitual ou hipotético são as palavras características para esse domínio nãocomportamental. Resumindo: o domínio da "mente". Versões diferentes de mentalismo utilizam termos diferentes para essas entidades inferidas.

O mentalismo também assume que as estruturas mentais inferidas a partir de domínios não comportamentais atuam como causas antecedentes para o comportamento. Eventos, variáveis e relações ambientais observáveis também antecedem o comportamento. Mas não seriam causas, de acordo com o mentalismo. $\mathrm{Na}$ melhor das hipóteses, estes serviriam de gatilhos para as estruturas subjacentes que causariam o comportamento. Assim, em primeiro lugar, assume-se que o organismo e seu comportamento estariam em contato direto apenas com as estruturas mentais e suas decorrências, e não com os fatores ambientais. Mas de que modo algo com o qual o organismo não estaria em contato direto pode ser tomado por causa? Em segundo lugar, o comportamento é mais diversificado e mais flexível do que uma explicação em termos de fatores ambientais possibilitaria. Considere, como um exemplo, a organização sequencial do comportamento. Tal comportamento não é uma mera cadeia de reflexos concatenados. O fluxo comportamental é mais rico e mais flexível do que isso. Assim, as variáveis independentes deveriam ser pelo menos tão ricas e tão flexíveis quanto a variável dependente, o que leva a crer que uma explicação em termos das propriedades causais de estruturas mentais subjacentes se faz necessária.

As propriedades funcionais das estruturas mentais causais nas diferentes versões de mentalismo variam. Ora são tidas como iniciadoras e ora como mediadoras. Por iniciadoras, nós dizemos que as próprias estruturas são consideradas como fontes do comportamento, de tal modo que nenhuma outra causa é necessária para explicá-lo. Por mediadoras, nós entendemos que uma ou mais estruturas são ativadas ou propulsionadas por estímulos externos 
observáveis. Essas estruturas mediadoras, ou intervenientes, que não são observáveis, estariam causalmente conectadas de um modo sistêmico e complexo, do qual uma resposta observável seria a consequência. Usualmente, as explicações mentalistas se assemelham à abordagem $\mathrm{S}-\mathrm{O}-\mathrm{R}$, que está presente na história do behaviorismo. $\mathrm{O}$ termo $\mathrm{O}$ faz referência explícita às variáveis "organísmicas", nãocomportamentais, inferidas, tidas como mediadoras das relações entre $S$ e $R$, e que fornecem a riqueza e a flexibilidade desejadas. Contudo, o mentalismo argumenta que seu poder explicativo é vastamente superior, porque seus mediadores mentais são vastamente superiores em comparação aos do behaviorismo.

De qualquer modo, as propriedades das estruturas mentais causais não podem ser caracterizadas nos mesmos termos e não funcionam de acordo com os mesmos princípios dos eventos observáveis, variáveis, e relações com o ambiente. Eventualmente, assume-se que as estruturas necessariamente produzem o conteúdo sobre o qual outras estruturas ou processos atuam, como uma analogia ao fluxograma de programas de computador. Mas, de novo, o papel de tais estruturas não é determinado nem materialmente e nem unicamente pela experiência de um organismo. O mentalismo se aplica quando tanto o domínio quanto suas estruturas causais são materialistas ou dualistas (i.e., dualismo metafísico, ou de substância).

Reconhecendo que diferentes formas de mentalismo concebem as propriedades causais das estruturas subjacentes de modo distinto, podemos notar que para o mentalismo a explicação causal do comportamento é - na melhor das hipóteses - incompleta, e - na pior das hipóteses - defeituosa, se utiliza apenas conceitos de domínios observáveis, tais como eventos ambientais, variáveis e relações, que são observáveis. Esclarecendo: um organismo experimenta eventos ambientais que são observáveis, variáveis e relações ao longo de sua vida; mas, para o mentalismo, estes fatores ambientais são relevantes apenas para a performance. Eles não são as causas em si. E a compreensão teórica e a explicação do comportamento que é apropriada para a ciência não pode ser oferecida em termos relativos a tais fatores.

Os mentalistas frequetemente defendem que seu ponto de vista está inserido na história da ciência. De acordo com eles, o progresso em uma ciência decorre de inferências analíticas teoricamente ricas e conceitos explicativos que não são observáveis, no lugar de análises e explicações para eventos, variáveis, e relações que são observáveis, porém limitadas. Considera-se como exemplos representativos os átomos, elétrons, teoria da célula, a teoria das doenças causadas por germes e metabolismo celular. Nada disso podia ser diretamente observado no momento em que se inferiu a sua existência pela primeira vez. O argumento mentalista é o de que casos como esses indicam porque a ciência não deveria se restringir aos eventos observáveis, variáveis e relações. Previsão e controle irão naturalmente aparecer como uma consequência de se ter feito inferências sobre as estruturas subjacentes e não observáveis que constituem o interesse genuíno da ciência, porém previsão e controle são tópicos típicos da engenharia. A psicologia cognitiva, de modo análogo às inúmeras formas de mentalismo, age do mesmíssimo modo que outras ciências genuinamente orientadas por teorias para inferir a existência de fenômenos subjacentes e não observáveis para, em seguida, tentar demonstrar a validade das inferências por meio da experimentação.

Por certo, o mentalismo endossa a posição de que a ciência busca explicações. Para o mentalismo, explicações são afirmações que oferecem as bases para a compreensão teórica dos mecanismos ou princípios que são postulados como subjacentes aos eventos que observamos. Um postulado epistemológico bastante tradicional no âmbito do mentalismo consiste em dizer que as explicações científicas começam com nossa observação, mas não terminam com ela. Ao contrário, as explicações científicas devem apelar para os fatores subjacentes que são parte de um domínio diferente daquele que é observável. Novamente, o argumento mentalista sustenta que a história da ciência sugere que progressos são obtidos graças ao olhar para além e por detrás daquilo que observamos, para então fazer afirmações acerca dos fatores causais subjacentes, que não foram diretamente observados. Na melhor das hipóteses, o estudo dos fatores observáveis de um evento é útil na medida em que provê embasamento que sustente as inferências sobre os fatores subjacentes, como as verdadeiras causas de um evento. Por isso, no caso do comportamento e de outros fenômenos psicológicos como a aprendizagem, a percepção, e a memória, as causas são as estruturas subjacentes que promovem a capacidade e a competência para se comportar em circunstâncias muito particulares. Logo, estudar a relação entre os fatores observáveis em um evento seria apenas descrever a performance e como o evento surgiu, e não explicar o porquê de seu surgimento.

De que modo, então, reconciliaríamos a visão de que as estruturas mentais subjacentes necessárias para a explicação não são observáveis, com a noção de que a ciência lida com fenômenos que são publicamente observáveis? Para o mentalismo, a questão não é tão problemática quanto parece ser. A ciência lida com coisas que não podem ser diretamente observadas ou mensuradas. No mentalismo, as estruturas mentais não observáveis podem ser operacionalmente definidas como construtos hipotéticos. Então, a ciência pode lidar com elas de forma indireta, no nível conceitual, por meio daquilo que é considerado como sendo manifestações das mesmas e, por conseguinte, oferecer explicações válidas. Neste particular, pesquisadores e teóricos estariam livres para postular a existência de quaisquer estruturas mentais que sirvam como explicações para um fato observável que é posto em questão e, então coletar dados que permitam verificar a adequação desses postulados. Para os mentalistas, o behaviorismo busca explicações que são limitadas ao domínio observável. Algo que é, seguramente, inadequado. O mentalismo seria mais rico e preferível porque admite conceitos explanatórios que são mais ricos e preferíveis, precisamente porque não estão restritos ao que é observável. 
Conforme sugerimos anteriormente, os mentalistas contrastam sua posição diretamente com o behaviorismo. De acordo com eles, os mentalistas, as explicações comportamentais são colocadas apenas em termos daquilo que é observável a partir dos eventos ambientais, variáveis, e relações $\mathrm{S}-\mathrm{R}$. Consequentemente, as explicações comportamentais dão enfoque somente na descrição da performance - "O que aconteceu?". Mentalistas asseguram que tais explicações são incompletas porque, no melhor dos casos, elas não especificam as estruturas causais subjacentes e não observáveis responsáveis pela performance. Em vez disso, explicações mais apropriadas devem responder questões do tipo "Por que isso aconteceu?", no sentido de "Qual estrutura subjacente é responsável pelo que aconteceu?". O ponto é que se a estrutura teórica subjacente não foi previamente postulada, o comportamento não foi verdadeiramente explicado. Por isso, o behaviorismo não pode ser considerado como gerador de conhecimento explanatório e teórico genuíno, e não pode ser considerado como sendo genuinamente científico.

De acordo com o mentalismo, todo mundo sabe que seres humanos possuem mentes que não são observáveis. Em sentido mais amplo, a mente pode ser compreendida como um conjunto de estruturas mentais que medeiam o comportamento em circunstâncias específicas. Como diz o ditado: a mente é aquilo que o cérebro faz. As propriedades funcionais dessas estruturas mentais são inatas e de modo algum influenciadas pela experiência. Caso o fossem, elas seriam comportamentais e descritivas, ao invés de mentais e genuinamente explanatórias. Qualquer explicação para o comportamento humano que afirma ser uma explicação que não leva em consideração o papel de estruturas teóricas não observáveis, tal como a mente, não é, seguramente, a mais adequada.

Explicações para o comportamento de animais não-humanos, como ratos e pombos de laboratório, ou de seres humanos com desenvolvimento atípico, não são necessariamente problemáticos em termos de estruturas teóricas não observáveis. Todavia, as explicações para o comportamento de seres humanos com desenvolvimento típico devem, sem sombra de dúvidas, apelar ao funcionamento de suas mentes. As explicações no campo da sociedade e da cultura também devem seguramente apelar para as mentes e as estruturas mentais subjacentes.

Em suma, o mentalismo defende que a mente é parte das explicações psicológicas, e que se pode lidar com a mente de uma maneira cientificamente aceitável.

\section{A VISÃO ANALÍTICO-COMPORTAMENTAL DE CIÊNCIA}

Como, então, a análise do comportamento se posiciona em relação a esses muitos assuntos? Claramente, uma das principais diferenças entre $o$ mentalismo e a análise do comportamento gira em torno das diferentes concepções da explicação causal do comportamento.
Começamos sugerindo que, para a análise do comportamento, a ciência é, em grande medida, o comportamento operante dos cientistas, juntamente com (a) os artefatos associados à origem e à execução de seu comportamento e (b) os artefatos produzidos por seu comportamento. Assim, o comportamento científico varia de atividades de pesquisa em andamento no laboratório até conversas e escritos sobre essas atividades. Os artefatos associados à origem e à execução de seu comportamento vão desde o aparato científico que usamos nas atividades de pesquisa, como tubos de ensaio, balanças, microscópios e assim por diante, até a base de dados estabelecida e métodos codificados de procedimentos científicos. Os artefatos produzidos por seu comportamento vão desde novos aparelhos científicos, como novas escalas e microscópios, até extensões de pesquisas publicadas, refinamentos de teorias, explicações alternativas e novas interpretações.

Além disso, os reforçadores para o comportamento operante da ciência caem em um continuum. De um lado está a previsão e o controle de eventos naturais. Nesse sentido, nos preocupamos em moldar a natureza como uma bigorna e obter resultados que nos reforcem por meio de nossa ação direta e prática. No outro extremo está a descoberta de uniformidades, a ordenação de dados confusos e a resolução da perplexidade. Nesse sentido, estamos preocupados com os produtos verbais generalizados e abstratos da ciência, como teorias, explicações e interpretações que transcendem instâncias particulares de predição e controle. Esses produtos abstratos são derivados de empreendimentos mais específicos e tornam-se progressivamente mais abstratos à medida que a ciência e a tecnologia progridem.

A ciência primitiva presumivelmente começou com tecnologias primitivas: fazer panelas de barro, fazer martelos e espadas, roupas, casas, meios de transporte, domesticar animais, criar seletivamente plantas e animais. As culturas então desenvolveram regras para transmitir o conhecimento adquirido sobre essas tecnologias para as gerações futuras. As regras foram formalizadas como fontes de controle discriminativo.

Com o tempo, as regras tornaram-se mais generalizadas e abstratas, com maior grau de liberdade para sua aplicação - diferentes tipos de argila para vasos, diferentes tipos de metais para martelos e espadas, diferentes fontes de materiais para roupas, diferentes maneiras de domesticar animais. O ponto importante é que o comportamento verbal da ciência recai sobre um continuum pragmático que varia de (a) regras de artesãos, (b) relações funcionais, (c) conceitos analíticos e explicativos de ordem superior, (d) teorias, leis, explicações e interpretações. Em uma ponta do continuum, a principal preocupação é a ação direta, prática e efetiva em casos particulares. No outro extremo, a principal preocupação é tatear ordem e uniformidade em um nível abstrato e generalizado, baseando-se em organizações de dados. Teorias e explicações podem ser entendidas como derivadas e ampliadas a partir de uma base de relações funcionais. $\mathrm{O}$ mero acúmulo de 
resultados não é mais valioso como uma teoria, do que um monte de pedras [é valioso] como uma casa (e.g., Poincaré). Os dados se tornam úteis quando organizados, de modo que possam informar resultados.

Uma preocupação importante aqui é a natureza das contingências que influenciam o comportamento científico. Até que ponto o comportamento científico é influenciado por contingências decorrentes de (a) operações e contatos com dados que resultam em ação efetiva com relação à natureza - como predição e controle, em oposição a contingências oriundas de (b) tradições sociais e culturais (c) processos linguísticos maliciosos, às vezes chamados de reificação, e (d) metáforas inapropriadas?

Outra maneira de avaliar a natureza das contingências que influenciam o comportamento científico é perguntar sobre os resultados para os quais o produto científico contribui. Estão seus resultados em um continuum pragmático que vai desde a previsão e controle de eventos particulares em uma extremidade, até a descoberta de uniformidades, a ordenação de dados confusos, e a resolução de perplexidade, na outra extremidade? A análise do comportamento endossa qualquer um desses tipos de contribuições. Alternativamente, são seus resultados uma questão de conformidade com declarações de revelação e autoridade, derivadas de um suposto domínio além do mundo natural e com consideração mínima ao resultado de interações com o mundo natural? Para a análise do comportamento, o mentalismo está comprometido com essa última orientação, e as práticas explicativas que ela promove são problemáticas.

Uma característica importante da visão acima é que, para a análise do comportamento, o comportamento científico é progressivo e contínuo, em vez de dicotômico. Isto é, as posições sistemáticas de grandes cientistas e teóricos como Galileu, Newton, Darwin, Watson e Crick, e Hawking, cresceram a partir de dados observacionais, próprios ou de outros. Um tipo de conhecimento científico não é concebido como uma mera tecnologia, preocupada com a aplicação ou engenharia, e outro tipo como um entendimento teórico, despreocupado com a aplicação e mutuamente exclusivo do primeiro tipo. O primeiro tipo não é concebido como um esforço um tanto rasteiro, e o segundo tipo como intelectualmente superior e, portanto, mais merecedor de estima e reconhecimento. Finalmente, a análise do comportamento não é concebida como uma instância a ser depreciada, do primeiro tipo, e o mentalismo como uma instância a ser reverenciada, do segundo tipo. Com certeza, diferentes formas de atividade científica podem ser encontradas em diferentes loci no continuum, mas uma consideração importante é que as formas são relacionadas, mais do que dicotômicas. Distinguir as formas, como faz o mentalismo, trai uma metafísica e uma epistemologia que se afastam de preocupações naturalistas e de uma compreensão do comportamento humano que produziu essas formas de atividade científica.

\section{IMPLICAÇÃO \#1: POR QUE OS CIENTISTAS FAZEM CIÊ̂NCIA?}

Por que então os cientistas fazem ciência? Para a análise do comportamento, fazer ciência é ocasionado por circunstâncias antecedentes particulares e mantido por resultados particulares. Sidman (1960) identificou várias razões pelas quais os cientistas conduzem pesquisas científicas: (a) para avaliar hipóteses; (b) satisfazer a curiosidade do investigador sobre a natureza; (c) experimentar um novo método ou técnica; (d) estabelecer a existência de um fenômeno; e (e) explorar as condições (limites) sob as quais um fenômeno ocorre. Note que algumas instâncias da ciência podem testar hipóteses, mas nem todas as ciências precisam fazê-lo. Assim, a pesquisa é uma atividade que produz conhecimento generalizável. O objetivo final é criar SDs verbais generalizados para interação efetiva com a natureza, mesmo que os produtos verbais sejam abstratos.

\section{IMPLICAÇÃO \#2: MÉTODOS DE PESQUISA}

Os métodos de pesquisa na análise do comportamento se preocupam em identificar manipulações que promovam uma ação direta e efetiva. Esses métodos isolam essas manipulações e seus efeitos por meio de várias técnicas (discriminação e reversão, sondas, linhas de base múltiplas, condições de controle etc.) que descartam possibilidades alternativas ou rivais. $\mathrm{Na}$ medida em que circunstâncias antecedentes ou características da população sejam relevantes, elas são então incorporadas conforme o necessário, por exemplo, para identificar os limites de classes.

Em contraste, os métodos de pesquisa na psicologia tradicional são baseados em estatísticas inferenciais e técnicas de amostragem. A questão é se uma observação particular é considerada o efeito metafísico da manipulação em questão, em oposição a simplesmente um erro amostral aleatório de uma população de resultados. Considerações pragmáticas, como a significância clínica e estatística do efeito, não estão normalmente envolvidas.

\section{IMPLICAÇÃO \#3: DEMARCAÇÃO DA CIÊNCIA E DA NÃO-CIÊNCIA}

Para os analistas do comportamento, a ciência está preocupada com o continuum pragmático, acima descrito, no domínio do mundo natural, e pode ser demarcada da não-ciência. Declarações não técnicas sugerem que a ciência (a) é guiada por lei natural, (b) explica eventos em termos da lei natural, (c) formula explicações que são testáveis por meio de observações, (d) oferece explicações que são mutáveis e provisórias, e (e) oferece explicações que são potencialmente falsificáveis, atentas às fontes de controle sobre o comportamento verbal científico que são encontradas no mundo natural. A não-ciência está preocupada com a revelação e a autoridade, derivadas de um suposto domínio além do mundo natural. Observe que a demarcação não é necessariamente dependente de a ciência ser ou não diretamente observada ou diretamente manipulada. Naturalmente, podemos buscar por explicações científicas de porquê os indivíduos fazem declarações fantasiosas e não científicas. 


\section{IMPLICAÇÃO \#4: CRITÉRIOS PARA PRÁTICA BASEADA EMPIRICAMENTE}

Os critérios para a prática baseada empiricamente no nível de um tratamento ou intervenção terapêutica bem estabelecida incluem tipicamente (a) pelo menos duas demonstrações de eficácia, ou uma série de delineamentos de caso único; (b) por pelo menos dois experimentadores diferentes; (c) usando uma intervenção documentada (por exemplo, por meio de um manual) com variáveis independentes e dependentes adequadas; e (d) seguindo um delineamento experimental adequado, com controles para fins de comparação. Na psicologia tradicional, esses critérios devem ser cumpridos por meio de um ensaio clínico randomizado, com delineamento do grupo e avaliação dos resultados por um teste de inferência estatística. Recentemente, uma ampla série de experimentos de delineamento de caso único que demonstram eficácia, com bom delineamento experimental, e resultados comparáveis a outro tratamento estabelecido, também foi considerada satisfatória. Esses critérios não são declarados de uma maneira totalmente coerente com uma orientação behaviorista radical. No entanto, pelo menos, vemos a preocupação com a confiabilidade e validade do comportamento verbal científico em termos das fontes de controle, como sugere a análise comportamental.

\section{SUMÁRIO E CONCLUSÕES}

Vimos que o mentalismo afirma que a análise do comportamento lida apenas com eventos e relações $S-R$ observáveis, ao passo que o mentalismo lida com estruturas teóricas subjacentes e inobserváveis. Consequentemente, o mentalismo argumenta que é cientificamente superior. Mais uma vez, essa afirmação é complexa. Por exemplo, a análise do comportamento reconhece eventos comportamentais privados, que, por definição, não são publicamente observáveis. Alguns desses eventos são verbais, como nos casos do pensamento e da resolução de problemas. A consideração importante aqui é que, embora esses eventos sejam privados, eles estão funcionalmente relacionados a circunstâncias ambientais na vida do agente, ao invés de serem inatos, como as ditas estruturas mentais. Dessa forma, a análise do comportamento reconhece variáveis independentes inobserváveis significativas em uma explicação do comportamento. Como resultado, as alegações de mentalismo são muito amplas e desinformadas.

Por mais importante que possa ser uma discussão acerca de variáveis independentes observáveis versus inobserváveis, talvez ainda mais importante seja uma distinção entre mentalismo e análise do comportamento, que se volte para suas respectivas concepções de comportamento verbal. Para o mentalismo, o comportamento verbal é um processo referencial e simbólico. Nesse sentido, palavras são símbolos que possuem significado. O significado é uma questão acerca daquilo a que as palavras se referem. Em alguns casos, o referente é publicamente observável. Em outros casos, o referente é inobservável - uma entidade mental. Para o mentalismo, no caso da psicologia, o referente mental é muitas vezes uma construção teórica na mente do cientista, que então precisa ser definida operacionalmente, de modo a alcançar concordância e evitar as armadilhas de abordagens introspectivas. Seres humanos são concebidos para serem organismos usuários de símbolos, e toda a abordagem supostamente segue essas suposições.

Em contraste, a análise do comportamento considera o comportamento verbal como uma forma de comportamento operante, passível de análise em termos de contingências. As contingências podem ser complexas, mas a análise permanece no nível das contingências. Não faz mais sentido dizer que um termo ou conceito é um símbolo que se refere a algo na mente do cientista, do que dizer que pisar no freio de um carro é um símbolo que se refere a parar na mente do motorista. Representações, e outras características do mentalismo, simplesmente não estão envolvidas. Atribuir o comportamento verbal ao uso de símbolos e processos referenciais obscurece a análise de forma inextricável. Como vimos, as questões centrais são a natureza do controle de estímulo e a natureza do reforço do comportamento operante chamado científico. $\mathrm{O}$ mentalismo frequentemente reifica conceitos de nossa linguagem cotidiana. $\mathrm{O}$ mentalismo ainda supõe que os conceitos assim reificados representam algum tipo de entidade ou processo mental discreto com relevância científica, mesmo que a entidade esteja em outro domínio.

A análise do comportamento sugere uma maneira diferente de abordar esses assuntos. Para a análise do comportamento, o processo verbal chamado de abstração é comportamental. A abstração diz respeito ao controle de estímulos que surge de um aspecto restrito do contexto anterior. Nenhuma suposição é feita de que (a) o aspecto nomeado representa alguma estrutura de outro domínio, (b) a ciência se preocupa com essa estrutura como eventos observáveis subjacentes, e (c) as observações permanecem como evidência para a existência da estrutura. Assumir isso leva a ciência a percorrer um longo caminho de interações ineficazes com o mundo.

A análise do comportamento está, em última instância, preocupada com o mentalismo em bases pragmáticas, e não ontológicas. Mais especificamente, a análise do comportamento argumenta que o mentalismo obscurece, e de fato impede ativamente, a busca de detalhes importantes sobre as relações genuinamente relevantes entre comportamento e ambiente, reduz a curiosidade ao nos levar a aceitar "ficções explicativas" fantasiosas como causas, deturpa os fatos a serem explicados e nos dá falsas garantias sobre o estado de nosso conhecimento. Consequentemente, a análise comportamental argumenta que o mentalismo interfere efetivamente na previsão, controle e explicação eficazes do comportamento, apesar das asserções mentalistas em sentido contrário. A concepção de variáveis mentais implica que elas e o comportamento que causam surgem e operam de forma relativamente independente das circunstâncias ambientais, e nada podemos fazer para promover formas benéficas de comportamento ou substituir formas problemáticas, certamente uma postura não-otimista para uma ciência da vida. Para a análise do comportamento, respostas genuínas sobre as causas e a 
explicação do comportamento podem ser encontradas na análise de contingências em nível de filogenia, ontogenia e cultura.

Termos e conceitos-chave: análise do comportamento, explanação/explicação, construtos hipotéticos, estruturas mentais, mentalismo, método científico, teoria, comportamento verbal.

\section{REFERÊNCIA}

Sidman, M. (1960). Tactics of scientific research. New York: Basic Books.

Submetido: $28 / 03 / 2018$

Aceito: $21 / 04 / 2018$ 